\title{
Planar C-Band Antenna with Electronically Controllable Switched Beams
}

\author{
Mariano Barba, Juan E. Page, and Jose A. Encinar \\ Departamento de Electromagnetismo y Teoria de Circuitos, Escuela Técnica Superior de Ingenieros de Telecomunicación, \\ Universidad Politécnica de Madrid, Ciudad Universitaria, 28040 Madrid, Spain \\ Correspondence should be addressed to Mariano Barba, mbarba@etc.upm.es
}

Received 14 October 2008; Accepted 8 January 2009

Recommended by Giovanni Toso

The design, manufacturing, and measurements of a switchable-beam antenna at $3.5 \mathrm{GHz}$ for WLL or Wimax base station antennas in planar technology are presented. This antenna performs a discrete beam scan of a $60^{\circ}$ sector in azimuth and can be easily upgraded to 5 or more steps. The switching capabilities have been implemented by the inclusion of phase shifters based on PIN diodes in the feed network following a strategy that allows the reduction of the number of switches compared to a classic design. The measurements show that the design objectives have been achieved and encourage the application of the acquired experience in antennas for space applications, such as X-band SAR and Ku-band DBS.

Copyright (C) 2009 Mariano Barba et al. This is an open access article distributed under the Creative Commons Attribution License, which permits unrestricted use, distribution, and reproduction in any medium, provided the original work is properly cited.

\section{Introduction}

The well-known features of planar antennas are low profile, low mass, potential low cost, and ease of mounting; they make this antenna technology very attractive for many communications applications of both terrestrial and space systems. Moreover, for space applications, their planar geometry is very interesting because the simplifications that its geometry introduces in the packaging for the launching and deployment systems.

On the other hand, the well-established printed circuit technology and the consolidated market of planar microwave devices allow the introduction of nonlinear components, such as PIN diodes, varactor diodes, and MEMS, which can be used to reconfigure or reshape the beam by controlling or changing the RF signals. In this way, the added flexibility to the antenna can give an agile response to the continuous changing needs in communication systems. A good review of the advantages of reconfigurable antenna for mobile communication systems can be found in [1]; these advantages are very similar to fixed wireless services such us wireless local loop (WLL) or Worldwide Interoperability for Microwave Access (Wimax). The ideal situation would be that one where a complete pattern reconfiguration is attainable in the antenna according to the system needs, in fact, this is achieved by using MIMO systems, however, they require many antennas, many antenna locations, and signal postprocessing. There is a very interesting way of developing agile antennas including partial and limited reconfigurability in just one single antenna by, as mentioned, the addition of controllable phase shifters in the antenna circuits at the RF level. Since base station antennas usually generate a pattern that covers a sector in azimuth with the appropriate beam shaping in elevation implemented by a linear array [2], the antenna can be designed to scan a sector typically covered by one passive antenna [3]. Alternatively, the antenna can be designed to switch the beam pointing direction to the area with a greater service demand. These solutions lead to electronically reconfigurable antennas at a moderate cost and complexity and very low power consumption. This concept of antenna reconfiguration is very suitable for space applications where switching, scanning, or reconfiguration of the beam is required to improve the performances of the system.

In this paper, a low-cost switchable antenna is proposed for base stations of WLL and Wimax systems in the $3.5 \mathrm{GHz}$ band. The objective of this development was to obtain a low-cost low-complexity antenna covering a $60^{\circ}$ sector with several switchable beams. A new topology is proposed for the beam switching, based on the implementation of 1 bit phase 


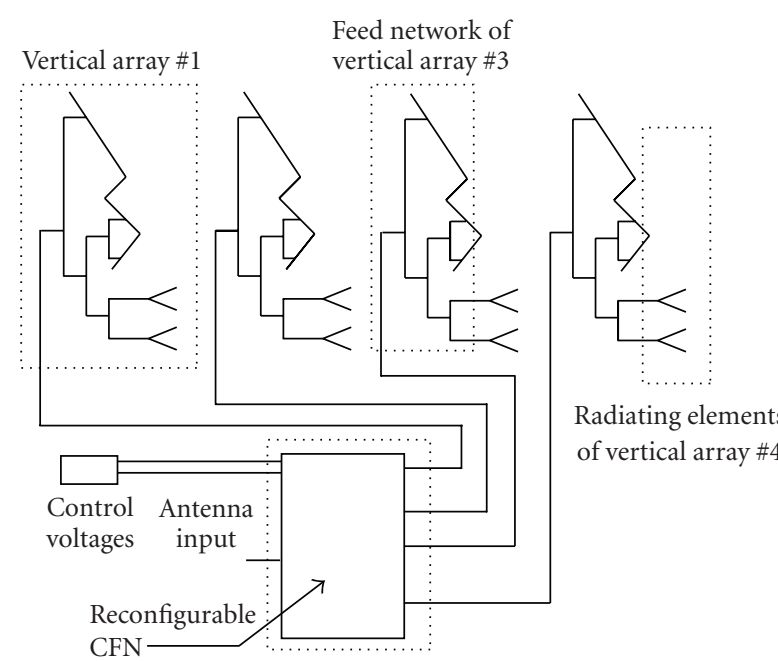

FIgURE 1: Antenna architecture.

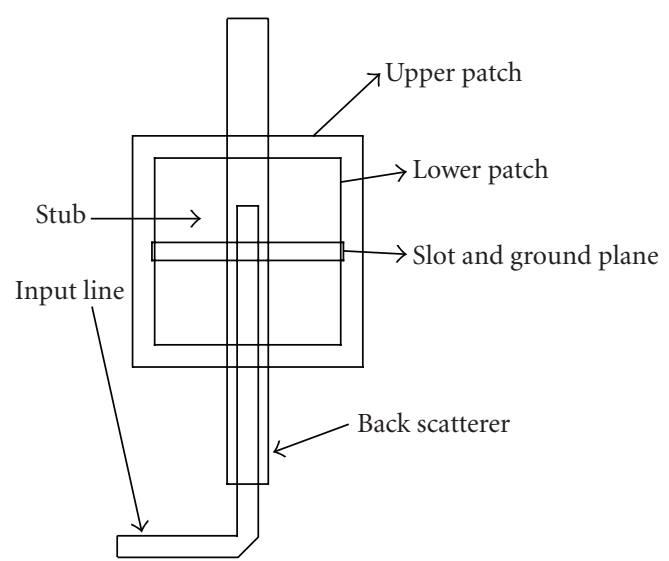

Figure 2: Radiating element. Top view and definition of conductors.

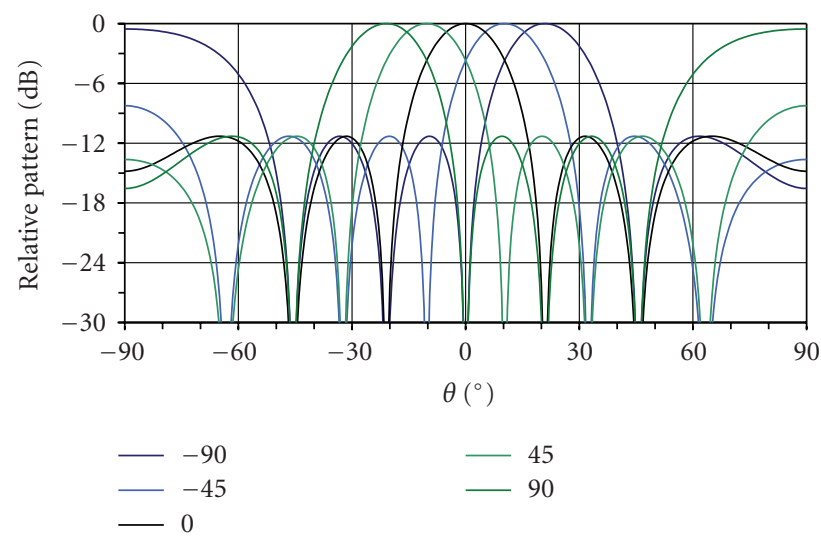

Figure 4: Five beams covering a $60^{\circ}$ sector.

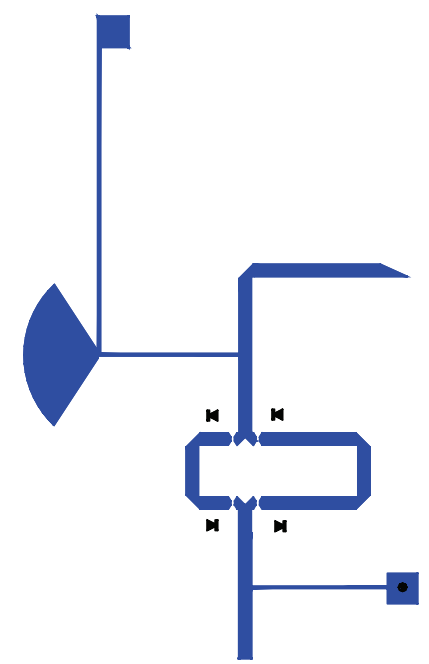

Figure 5: PIN diodes and microstrip delay lines phase shifter module.

shifters at each level of a conventional corporate feeding network. The distributed location of phase shifters in the feeding network yields a simplification of the antenna architecture. The concept of the proposed switchable antenna is demonstrated by the design, manufacture, and measurements of a prototype with three beams.

The development presented in this paper was focused on the scanning technology, circuits, and concepts to be applied to Wimax-WLL systems, but bearing in mind the use of the knowledge to develop switchable and reconfigurable antennas for other applications. In particular, the use of the concepts developed in this antenna for space systems is under consideration and most of the experience acquired with this development will be directly applied to other designs in progress for X-band SAR and Ku-band DBS applications.

\section{Antenna Description}

The antenna concept for the base station antenna with switchable beams addressed in this design is detailed in Figure 1. It is made up of a horizontal array of vertical 


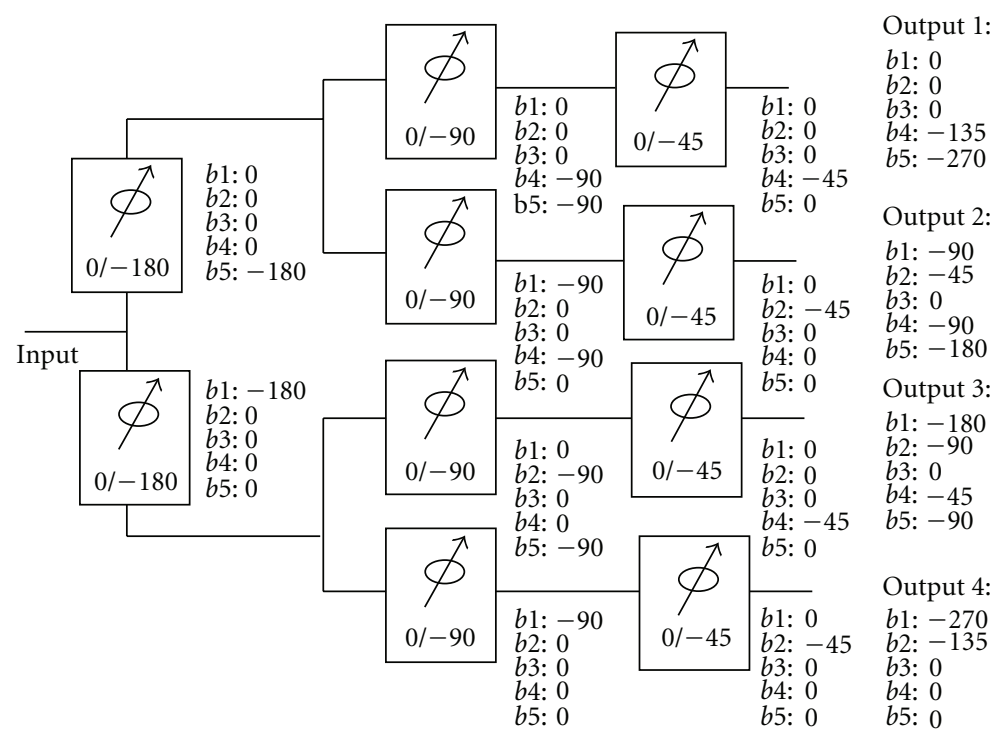

Figure 6: Phase shifter configuration and locations to obtain the desired beams.

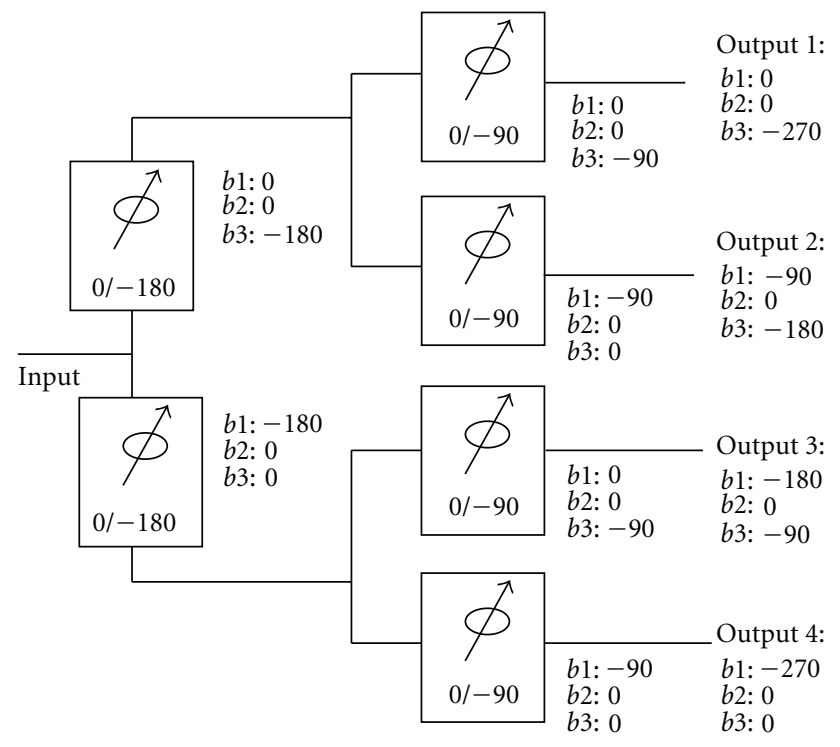

Figure 7: Phase shifter configuration to obtain 3 beams, the two extreme and the central.

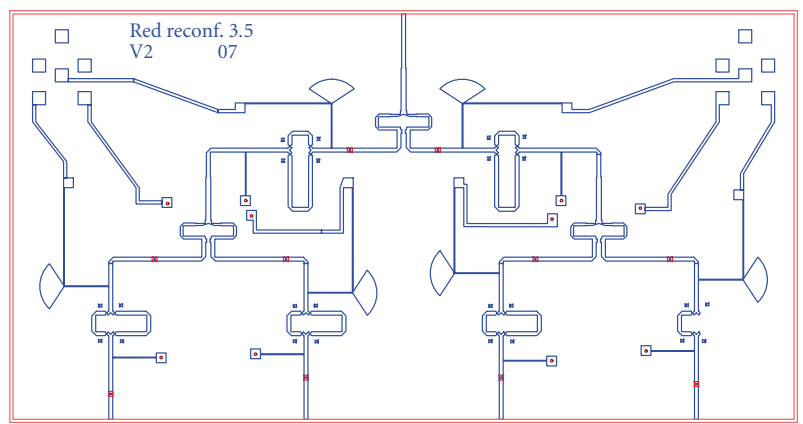

FIGURE 8: Drawing of the switchable feeding network. subarrays. The vertical subarray produces the desired elevation pattern. For base station antennas, the requirements for the elevation patterns typically include shaping for upper lobe reduction, and often squared cosecant and null filling. According to these requirements and the gain specifications, these arrays have at least 8 elements, but sometimes up to 12 or 14 elements. In the antenna presented in this paper, the horizontal array has been implemented to produce the desirable beam scanning in azimuth by the inclusion of the proper electronically controllable feed network, which gives the different feed distributions needed. In this work, the main objective was to obtain the demonstrator of the switchable-beams antenna. Therefore the vertical array was obtained with just two radiating elements with equal amplitude and a phase shift to obtain a $2^{\circ}$ degree tilt in the elevation pattern. The option for several elements in the vertical array is indicated by the saw lines in Figure 1. The reader is referred to $[4,5]$ for more discussions on the synthesis and the implementation of elevation beam-shaped patterns.

2.1. Radiating Elements. The radiating element is a dualstacked patch coupled to a microstrip line through a slot. It has been designed to obtain the sufficient bandwidth and matching level for the required frequency band in the final antenna. Its design objectives, band from $3.3 \mathrm{GHz}$ to $3.7 \mathrm{GHz}$, matching level better than $-22 \mathrm{~dB}$, have been established considering that this element has to be included in the array as its radiating element as well as considering the performance degradation that will be found in the prototypes as a result of the tolerance effects. This element is very similar to the one used in [3] for a lower frequency band. For reader convenience, their details and geometry are included in this paper; however, to focus this paper on the most relevant issues, the discussions about its design are avoided. Figure 2 shows the geometry including the 
TABLE 1: Definition of the lay-up for the radiating element.

\begin{tabular}{|c|c|c|c|c|}
\hline \multirow{2}{*}{ Description } & \multirow{2}{*}{ Type } & \multicolumn{2}{|c|}{ Dielectric layers } & \multirow{2}{*}{$\begin{array}{c}\text { Conductors } \\
\text { SIZE } \mathrm{mm} \times \mathrm{mm}\end{array}$} \\
\hline & & $\mathrm{H} \mathrm{mm}$ & DK & \\
\hline Upper patch & Conductor & & & $19.83 \times 19.83$ \\
\hline Upper substrate & Dielectric & 12 & 1.6 & \\
\hline Lower patch & Conductor & & & $24.64 \times 24.64$ \\
\hline Lower substrate & Dielectric & 6 & 1.6 & \\
\hline Ground plane & Conductor & & & Slot: $20.42 \times 1.55$ \\
\hline RF substrate & Dielectric & 0.5 & 3.2 & \\
\hline Microstrip, stub & Conductor & & & $w=1.9 ;$ stub $=4.73$ \\
\hline Air & & 15 & & \\
\hline Back scatterer & Conductor & & & $4 \times 50$ \\
\hline FR4 & Dielectric & 1.5 & 4.1 & \\
\hline
\end{tabular}

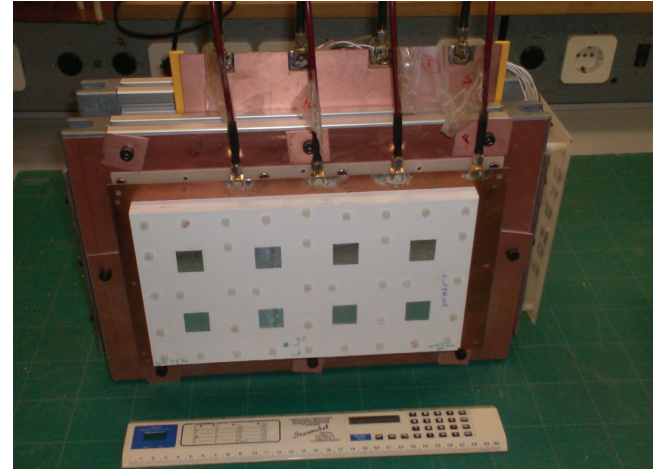

Figure 9: Phase antenna prototype. View of the radiating element and vertical subarray inputs.

Wimax $3.5 \mathrm{GHz}$.

Pp. 2, four vertical inputs.

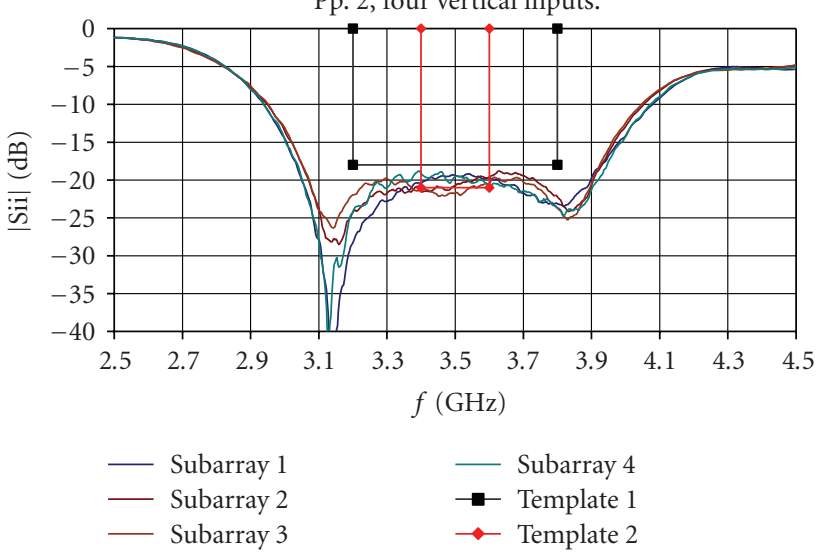

FIGURE 10: Antenna subarrays. Return losses for each input.

conductors of the radiating element and their description. In Table 1, the stack of the antenna and the parameters of the elements of the radiating element are listed.

The chosen separation distance for all of the radiating elements of both arrays is $60 \mathrm{~mm}(0.7 \lambda)$. This value has been taken as the maximum distance between the elements, where

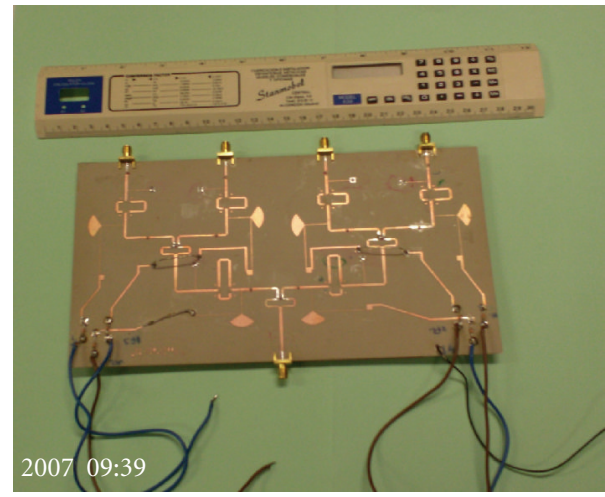

Figure 11: Prototype of feed network of the switchable antenna.

the disturbance introduced by the grating lobes level is not significant for the system requirements.

As has already been mentioned, the vertical array for this prototype consists of two elements fed with equal amplitude and with a phase shift that produces an elevation tilt of $2^{\circ}$. Figure 3 shows the arrangement of the vertical array for this prototype.

2.2. Feeding Network. The azimuth feed network has to provide switchable beams covering a sector of $\pm 30^{\circ}$. This can be done with an array of four elements, fed with equal amplitude and a progressive phase shift to tilt the pointing direction as desired. The desired coverage assuming a ripple of $2 \mathrm{~dB}$ can be obtained by 5 different beams pointing alternatively to $-21^{\circ},-10.5,0^{\circ}, 10.5^{\circ}$, and $21^{\circ}$, as shown in Figure 4 . To obtain these beam inclinations and considering the separation between elements, the feed network should provide five different feed distributions with a progressive phase shift between adjacent elements of $90^{\circ}, 45^{\circ}, 0^{\circ},-45^{\circ}$, and $-90^{\circ}$, respectively, and equal amplitude for all of them.

The feeding network has been designed and manufactured in microstrip technology. Although for this demonstrator, it is implemented in an independent board to allow all of the antenna components to be measured separately, however, in an industrial design, it can be integrated into the 

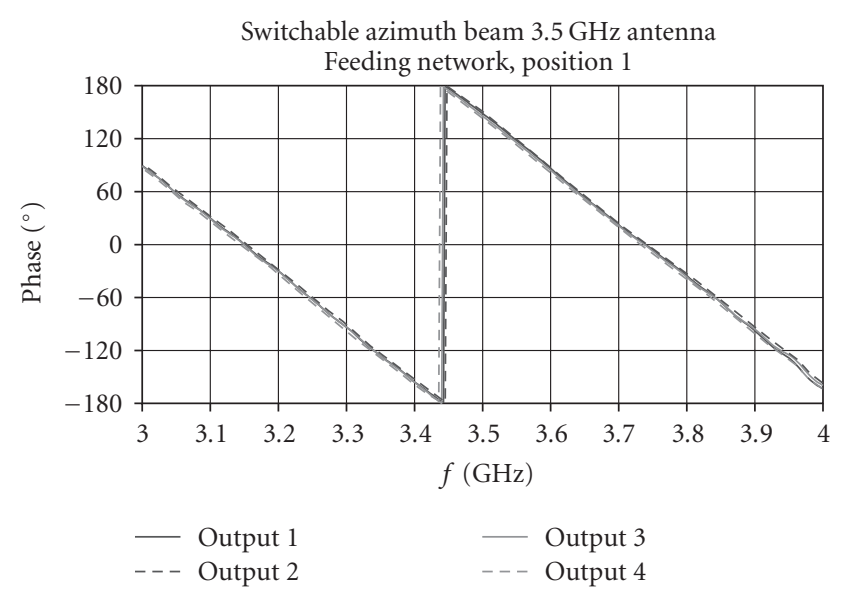

(a)

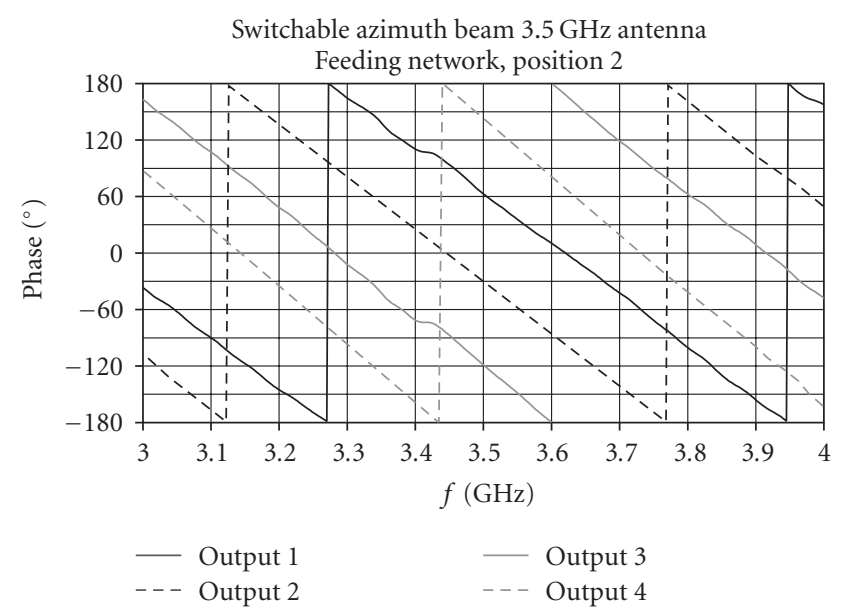

(b)

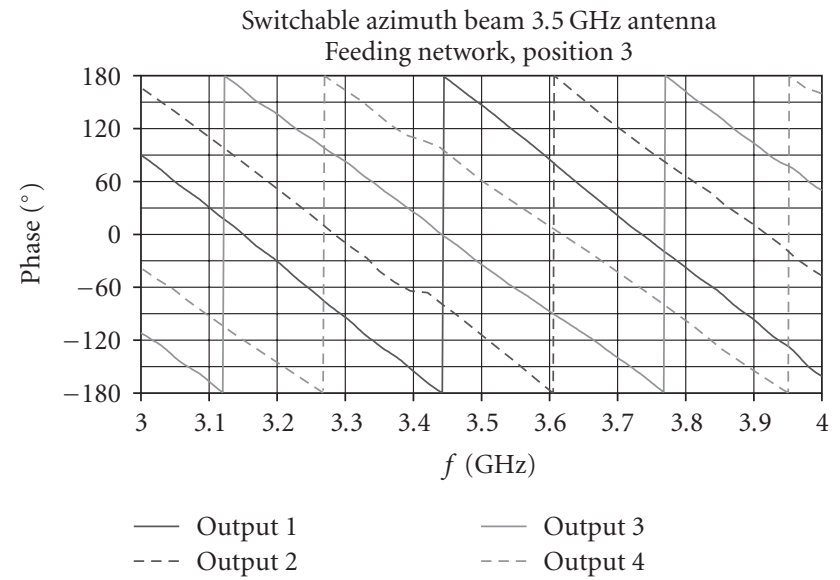

(c)

FIGURE 12: (a) Feed network measurement: output phases for position 1, (equal phase). (b) Feed network measurement: output phases for position $2,\left(-90^{\circ}\right.$ progressive phase shift). (c) Feed network measurement: output phases for position $3,\left(+90^{\circ}\right.$ progressive phase shift).

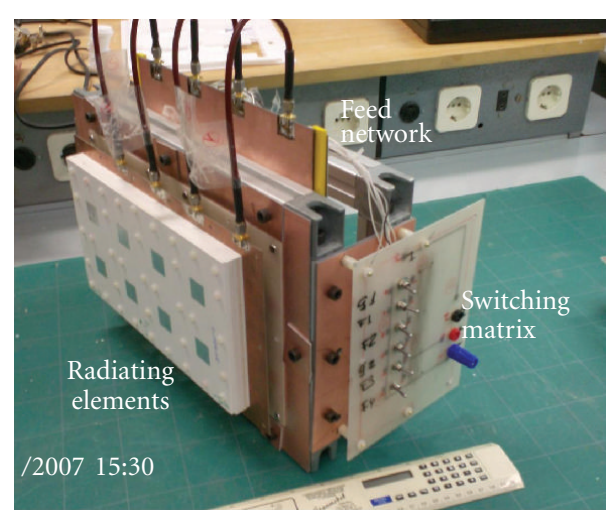

FIGURE 13: Photograph of the complete antenna.

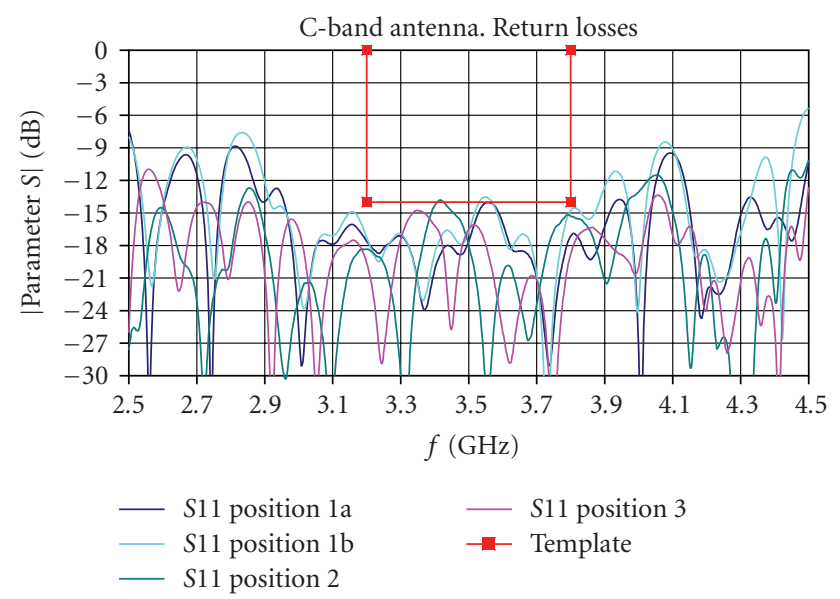

Figure 14: Complete antenna return losses at each pointing position.

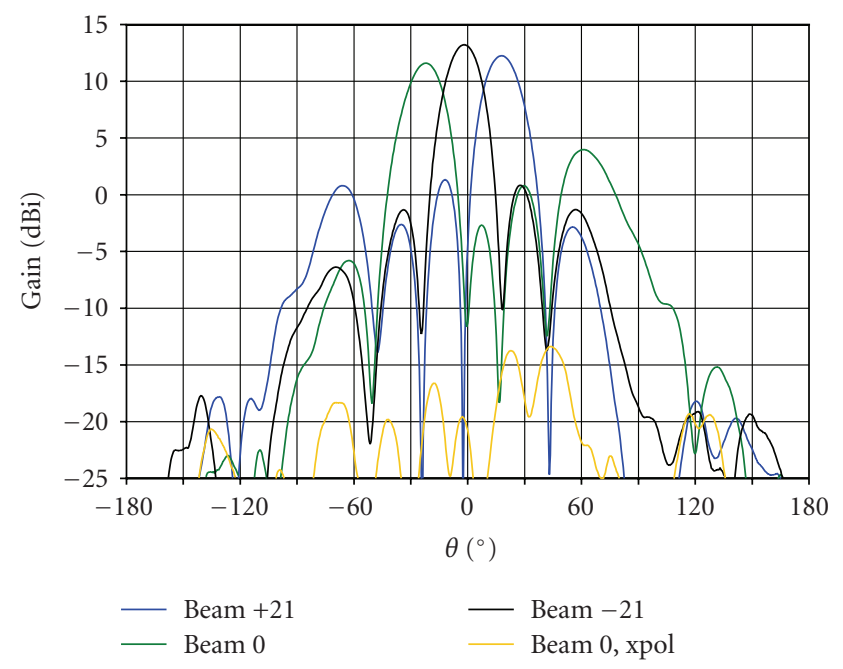

FIGURE 15: Antenna radiation pattern measurements. Azimuth cut for the three pointing positions. 


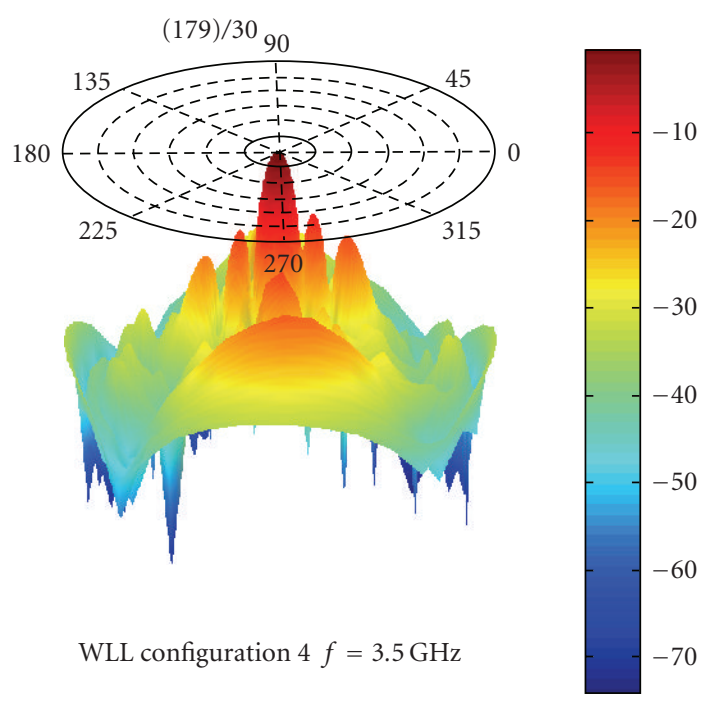

(a)

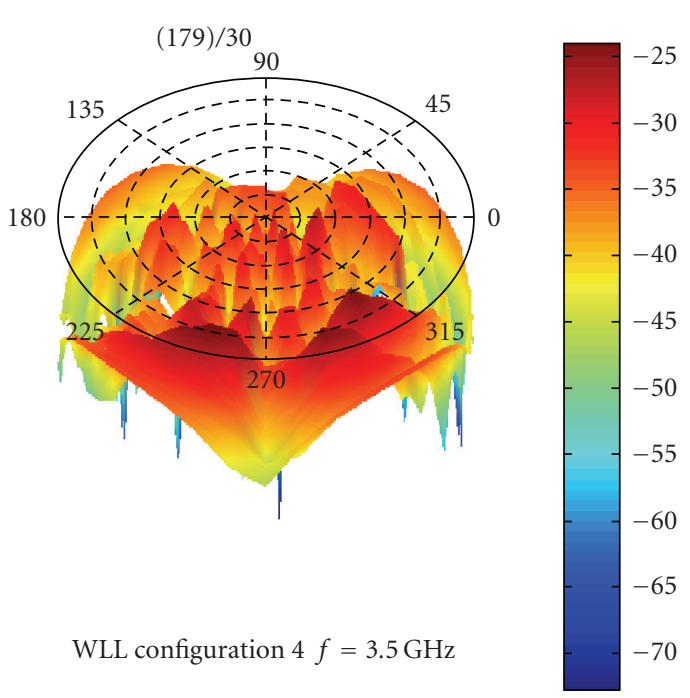

(b)

Figure 16: Pattern measurements. Three-dimensional pattern at $0^{\circ}$ pointing direction. (a) Copolar patterns, and (b) cross-polar patterns.

same board where the input microstrip lines of the radiating element are located, thus reducing the size, cost, losses, and complexity. The amplitude distribution can be obtained through a corporate feed network with equal amplitude dividers.

The phase shifter can be implemented by microwave switches single-pole double-through (SPDT) and delay lines, having each phase shifter two switches and two delay lines with the proper difference of length to produce the desired phase shift at the centre frequency. In this design, the SPDT switches have been implemented using two PIN diodes, resulting in the phase shifter module shown in Figure 5. The selected diode for this application that, according to manufacturer specifications, can be used at higher frequencies is MACOM-MA4PBL027. It presents an equivalent series resistance in direct biasing of $3.5 \Omega$ and an equivalent reverse capacitance lower than $40 \mathrm{fF}$.

The simplest way to implement the feeding network using amplitude dividers and two-state phase shifters consists of, first, obtaining the four equal amplitude signals, and after that division and before the inputs of each subarray, including a set of phase shifters connected serially, in order to implement the required additional phase shift states. However, this structure can be simplified by taking advantage of the corporate structure by including the phase shifter for more significant shifting in the first branches of the feed network. In Figure 6, this concept to implement the phase shifting needed for this design is presented. This figure also shows the setting of each phase shifter to obtain the 5 beams.

In order to develop the prototype and to reduce the manufacturing costs, the number of beams for the demonstrator was reduced to three, considering the extreme phase shiftings $\left(-90^{\circ}, 0\right.$, and $\left.+90^{\circ}\right)$. This option reduces the number of phase shifters and the manufacturing process, proves the concept keeping the most exigent beam tilts. Therefore, the configuration taken for the 3-beam demonstrator is the one set out in Figure 7.

For the $0^{\circ}$ beam pointing direction, only the situation with $0^{\circ}$ phase shift has been indicated. However, it is clear that other configurations of the phase shifter positions lead to the equal phase distribution. Any setting of the phase shifters with equal phase shifting at each level leads to an equal phase distribution and therefore could be valid.

The drawings of the design of the 3-beam feed network are shown in Figure 8, where it can be seen how the $180^{\circ}$ phase shifters are at one level of the corporate network and the $90^{\circ}$ phase shifters at the element (subarray input) level. All of the elements of the network such as bends, dividers, and biasing stubs have been optimized to reach the best performances in the band of interest 3.3-3.7 GHz. Even with this careful design, there are some parasitic effects introduced by connections of the diodes to the lines and through the connections of the blocking capacitors. In some conditions, as discussed before, these effects will be added each to others and can mainly degenerate the signal at the outputs. To reduce this degradation it is essential to implement the amplitude division with Wilkinson dividers, whose topology can be easily seen in Figure 8. High-quality microwave with low-loss blocking capacitors are needed; in this design, ATC series 600 were used.

\section{Prototypes and Measurements}

3.1. Antenna Components. Figure 9 shows the front image of the manufactured prototype. The eight radiating elements corresponding to the four subarrays can clearly be seen. The inputs of the four subarrays are also seen, as well as the cables which connect the outputs of the feed network to these inputs. In an industrial model, as it has been mentioned, the cable will not be used, since the feed network and the 
radiating element input lines can be integrated into the same board. However, at the prototype level they have been manufactured independently to allow the measurement of all of the elements and their validation.

The measured return losses at each subarray input are shown in Figure 10. It can be seen that their values are in range of $20 \mathrm{~dB}$ in the frequency of operation as it was expected from the simulations carried out during the design.

The manufactured prototype of the electronically controllable feeding network of the antenna is shown in Figure 11.

The set of measurements of the feed network is quite wide. The minimum needed are the reflection parameter and the transmission parameters (amplitude and phase) for the 3 feed distribution configurations. Because of space considerations, some of them are not included in this paper; the measured return losses are better of $-15 \mathrm{~dB}$ and the amplitude of transmission is in the range of $-9 \mathrm{~dB}$, giving, therefore, an average losses of the network of $3 \mathrm{~dB}$, where $1.5 \mathrm{~dB}$ are a result of the losses and another 1.5 to the RF microstrip lines.

In Figure 12, the output phases for the 3 configurations are shown. Figure 12(a) shows the measured output phases for the $0^{\circ}$ phase shift configuration, showing that the four signals are in phase. Figures 12(b) and 12(c) show the measured output phases for the $+90^{\circ}$ and $-90^{\circ}$ progressive phase shifts. These measurements confirm that the feed network is working properly.

3.2. Complete Antenna. Figure 13 shows a photograph of the complete antenna after assembling all of the elements, including a simple but efficient switching control matrix, which allows the phase shifters to be biased at their proper position according to the desired phase shifting at each configuration.

Figure 14 shows the measurement of the return losses for the 3 pointing positions with two options for the $0^{\circ}$ configuration. It can be seen that its value matches the typical specification for this type of antennas, $-14 \mathrm{~dB}$, which is equivalent to a VSWR of 1.5 .

Figure 15 shows the azimuth cuts in a plane of $2^{\circ}$ tilt for the 3 pointing directions of the far field measurements. It can be seen how beam switching is achieved as expected. As reference, the cross-polar measurement of the azimuth cut for the $0^{\circ}$ beam pointing direction is also included. It can be observed that its maximum level is lower than $25 \mathrm{~dB}$ below the antenna gain.

For reference, the three-dimensional measurements for co- and cross-polar components normalized to the maximum of radiation for the $0^{\circ}$ beam pointing direction are presented in Figure 16. The maximum level of cross-polar component in the range of the main lobe (from $-15^{\circ}$ to $+15^{\circ}$ ) is lower than $25 \mathrm{~dB}$ below the antenna gain.

\section{Conclusions}

This paper presents the design, manufacturing, and measurements of a switched-beam antenna at $3.5 \mathrm{GHz}$ in planar technology that demonstrate the suitability of scanning beam base station antennas for WLL and Wimax, where the beam scanning is achieved through the inclusion of phase shifters based on PIN diodes in the feed network, and where the strategy of phase shifter locations leads to a reduction in the number of components. The feasibility of this type of antenna for space applications is under consideration and the experience acquired with this development can be used for X-band SAR applications and Ku-band DBS.

\section{Acknowledgment}

This work has been supported by MEC, Spain, under Projects TEC2004-02155 and TEC2007-63650.

\section{References}

[1] L. C. Godara, "Applications of antenna arrays to mobile communications-part I: performance improvement, feasibility, and system considerations," Proceedings of the IEEE, vol. 85, no. 7, pp. 1031-1060, 1997.

[2] J. R. Sanford, J. F. Zürcher, and S. Robers, "Shaped beam patch arrays for mobile communication base station," Microwave Engineering Europe, pp. 31-33, June-July 1991.

[3] ETSI, "Fixed radio systems; electronically steerable antennas, Multipoint (MP) antennas; Fixed Wireless Access (FWA) radio systems," Tech. Rep. TR101938 VI.2.1, ETSI, Sophia Antipolis, France, 2002.

[4] M. Barba, J. E. Page, J. A. Encinar, and J. R. Montejo-Garai, "A switchable multiple beam antenna for GSM-UMTS base stations in planar technology," IEEE Transactions on Antennas and Propagation, vol. 54, no. 11, pp. 3087-3094, 2006.

[5] E. Carrasco, M. Arrebola, J. A. Encinar, and M. Barba, "Demonstration of a shaped beam reflect array using aperturecoupled delay lines for LMDS central station antenna," IEEE Transactions on Antennas and Propagation, vol. 56, no. 10, pp. 3103-3111, 2008. 

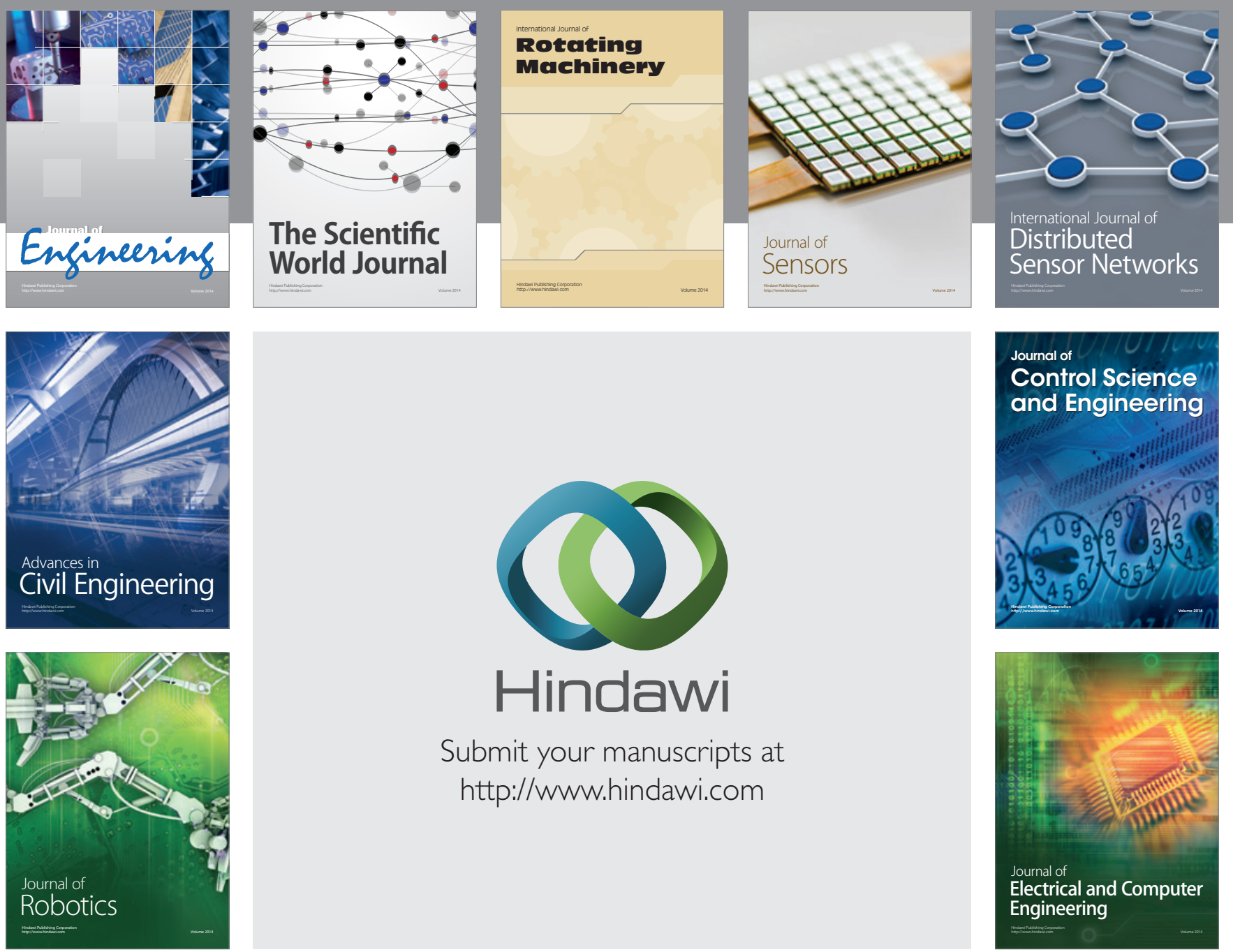

Submit your manuscripts at

http://www.hindawi.com
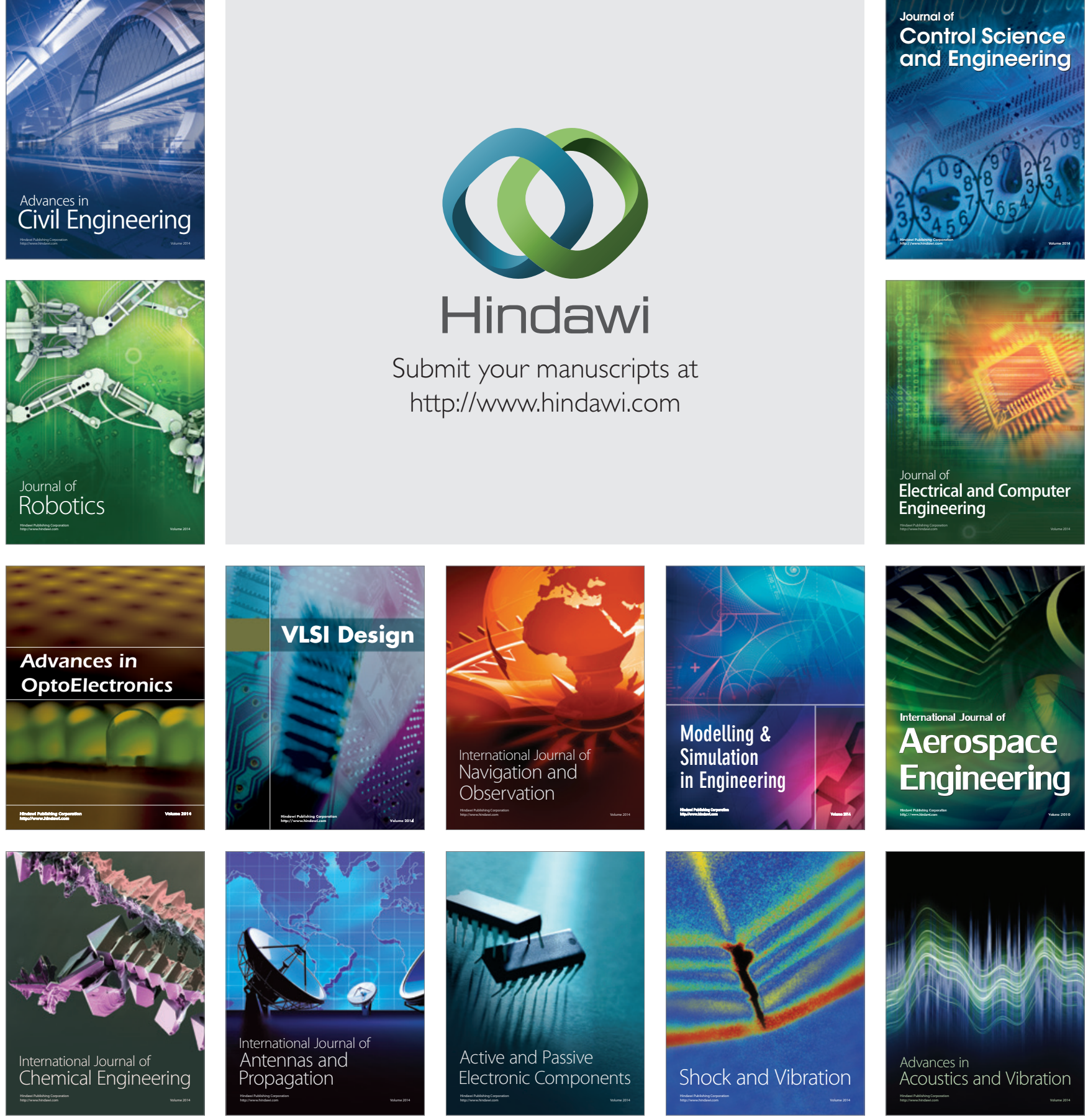